\title{
ESTIMATION OF INTERSECTION TURNING MOVEMENT FLOWS WITH THE TMERT3 MODEL VERSION: SENSITIVITY TO A WIDESPREAD DETECTOR FAILURE
}

Jelena Karapetrovic ${ }^{1}$, Peter T. Martin ${ }^{2}$

${ }^{1,2}$ Department of Civil Engineering, New Mexico State University, Las Cruces, NM, 88001, USA

Received 19 January 2021; accepted 19 March 2021

\begin{abstract}
Urban congestion is getting worse, especially at intersections, where conflicting flows share the same traffic signal cycle. However, traffic demand-responsive control can improve urban traffic operations by directing vehicles to alternative routes with spare capacity. Intersection demand is characterized by left, through and right turning movement (TM) flows from each intersection approach. Information on TMs must be current and consistently reliable for traffic control strategies to be effective. Non-recurring traffic congestion can quickly and unpredictably develop, such as during the Christmas shopping season, causing backups and warranting congestion mitigation. The latest version of the Turning Movement Estimation in Real-Time (TMERT) model, TMERT3, can consistently estimate 5-minute TM flows from $15 \%$ of network flow detections. This paper validates TMERT3 by showing it robustly estimates TMs despite widespread detector failure when applied to non-recurrently congested extensive networks. TMERT3 is tested on traffic on the 28-intersection network in Orem/Provo, Utah, USA, during a Christmas shopping season. TMERT3 TM estimates showed to preserve accuracy (Root Mean Square Error- RMSE) even when $20 \%$ of the initial detectors fail. So, TMERT3 presented practical applicability in efficiently guiding strategies that mitigate urban traffic congestion.
\end{abstract}

Keywords: Traffic State Estimation, the TMERT3 model version robustness, NETFLO algorithm, turning movement estimation, intersection control, congested network, Christmas shopping season, widespread detector failure, non-recurrent congestion

\section{Introduction}

Traffic congestion occurs when traffic demand approaches or exceeds the available roadway capacity, resulting in lower speeds, longer travel times, and increased queuing. Overall congestion is getting worse due to increased vehicle ownership and mobility. At this rate, around 20 million additional vehicles will use the US roads by 2025 (Vespa et al., 2020). Moreover, vehicle miles traveled are growing $1.1 \%$ annually (Leard et al., 2019). So, each US commuter in 2025 will waste $14 \%$ more time, $19 \%$ more money, and $9 \%$ more fuel than in 2017, due to increased congestion (Schrank et al., 2019).

Building infrastructure to accommodate the maximum demand is often impossible, expensive, or impractical as facilities can

${ }^{1}$ Corresponding author: iskra@nmsu.edu 
remain vastly underutilized during the off-peak. However, traffic management strategies guided by reliable and timely traffic demand information can efficiently reduce congestion as it develops (NCHRP, 2008). Spare capacity is a link capacity minus the corresponding traffic demand. A traffic control center can divert traffic from congested streets by guiding vehicles to spare capacity routes using variable message signs, traffic control signals, or in-vehicle devices.

Intersections are the main urban traffic system bottlenecks (Rao and Rao, 2012). Turning movement (TM) flows, associated with different maneuvers made from an intersection approach, characterize the traffic demand at urban intersections. An intersection configuration dictates the turning options. For example, demand at a typical four-legged intersection is identified through twelve TM counts, as vehicles could proceed through or turn left or right from each approach.

Manual collection of TM flows is not feasible, especially when traffic demand is high. However, video image processors identify TM flows from intersection camera images by analyzing successive image frames' changes (Yang and Pun-Cheng, 2017). Vehicle occlusion, illumination, or weather can corrupt the reliability of recorded TM flows, but motion trajectories recorded through GPS or lasers supplement and improve the measurements (Shirazi and Morris, 2016). While video image detection can offer reliable TMs, many agencies lack the resources to deploy video to all intersections. So, comprehensive realtime flow detectors are rare even at the most instrumented networks.

Agencies are often inclined to use the data already available to manage urban traffic congestion. Inductive loops are the USA's primary sensors that measure traffic flow between intersections (mid-block traffic) (Gordon and Warren, 2005). Multiple intersection TM arrangements can explain a set of complete mid-block detections, so models estimate the most probable one. Estimation models are inexpensive and can closely match actual TM flows from intersection stop-lines (Ghods and Fu, 2014; Riouali et al., 2019).

In reality, networks are rarely completely covered with detectors, seldom making TM estimates unreliable (Lan, 2001; Tuydes-Yaman et al., 2015). TM estimates can improve when complementing sparse detections with historical data, such as turning ratios (Mirchandani et al., 2001), origin-destination matrix ( $\mathrm{Wu}$ and Thnay, 2001), joint trip distribution (Nakatsuji et al., 2004), or route choice (Chen et al., 2012).

Still, a model might be non-transferable and produce unreliable TM estimates when socio-economic characteristics change (Mozolin et al., 2000; Zhai and Ardian, 2020). Also, these supplemental data are expensive and unavailable to most small agencies. So, agencies need a robust and inexpensive algorithm that can produce near real-time TM estimates from limited input and no historical data, such as TMERT.

\subsection{Model Description}

The Turning Movement Estimation in Real-Time (TMERT) model constraints the NETFLO algorithm to update TM flow estimates every 5 minutes from a mathematically represented network and a sparse set of mid-block detections (Martin, 1997). A modeled network allows taking a successive road link (arc) at a node. Each 
arc has an externally loaded node at both ends to account for vehicles entering and leaving the network borders or in-network parking lots. Taking an arc equates to driving through a road section or turning, and arcs differ through assigned constraints- weights and flow limits.

The NETFLO algorithm (Kennington and Helgason, 1980) minimizes the total travel cost on a network, so the objective function is a sum-product of arc flows and weights (Eq. 1). Three linear equations constrain flow generated at nodes and arcs. First, flows are accounted for either at arcs or nodes at all times (Eq. 2). Second, the total node external loading is zero for a standalone network (Eq. 3). Third, arc flows are within non-negative lower and upper bounds (Eq. 4).

$\min \rightarrow \sum w_{j} x_{j}, \forall j \in[1, m]$
Subject to:

$\sum x_{j}($ outflow $)-\sum x_{j}($ inflow $)=b_{i}, \forall i \in[1, n]$

$\sum b_{i}=0, \forall i \in[1, n]$

$0 \leq l_{j} \leq x_{j} \leq u_{j}, \forall j \in[1, m]$

Where: $w_{j}$ - weight per unit flow through arc $j, x_{j}$-flow through arc $j, l_{j}-$ arc $j$ lower bound, $u_{j}$ arc $j$ upper bound, $b_{i}$ - external loading at node $i, n$-number of nodes, $m$ - number of arcs.

Figure 1 presents arc types modeled in TMERT: detector (D), non-detector (ND), error (E), and TM. Detector arcs $x_{j}(D)$ are network links equipped with a midblock detector, while all other arc flows are estimated. TM arc flow estimates are used in assessing the model and represent intersection left, through, and right turns.

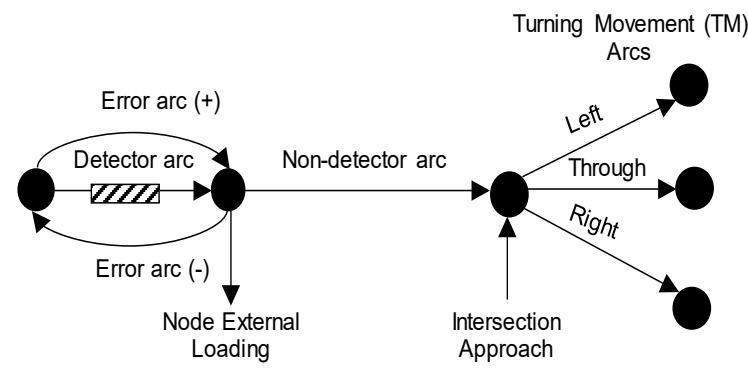

Fig. 1.

Arc Types

Arc flows are positive integers $\left(x_{j} \in Z_{0}^{+}\right)$, constrained through a set of non-negative integer lower $\left(l_{j} \in Z_{0}^{+}\right)$and positive-integer upper bounds $\left(u_{j} \in Z^{+}\right)$and non-negative integer weights $\left(w_{j} \in Z^{+}\right)$. So, the cost of taking an arc is $w_{j}$ and flow estimates iterate within a preset range $\left[l_{j}, u_{j}\right]$. Detected flows match lower and upper bounds $\left(l_{j}(D)=x_{j}(D)=u_{j}(D)\right)$ . This rigidity prevents the model from reaching a feasible solution $\left(x_{j} \in \Omega\right)$, but a pair of error arcs carrying imaginary flow permits the necessary flexibility. Non-detector and $\mathrm{TM}$ arc upper bounds $\left(u_{j}(N D), u_{j}(T M)\right)$ are found based on estimate procedures from the Highway Capacity Manual (HCM, 2010).

The base model, TMERT, lacked the comprehensive constraint regime that would 
yield TM estimates closely matching the observations from stop-lines. The three model versions: TMERT1, TMERT2, and TMERT3, improve TM estimates' consistency and accuracy through successively added model constraints. Each model version presented repeatability and transferability. Table 1 lists networks used in model calibration and validation for each model version.

Table 1

Calibration and Validation Networks Used in Modeling TMERT1, TMERT2, and TMERT3

\begin{tabular}{|c|c|c|}
\hline Model Version & $\begin{array}{c}\text { Calibration Network } \\
\text { (\# of modeled intersections) }\end{array}$ & $\begin{array}{c}\text { Validation Network } \\
\text { (\# of modeled intersections) }\end{array}$ \\
\hline TMERT1 & The Leicester, England (6) & The San Luis Obispo, California (20) \\
\hline TMERT2 & The Theoretical Network (15) & The Orem, Utah (11) \\
\hline TMERT3 & The Salt Lake City, Utah (8) & The Orem, Utah (11) \\
\hline
\end{tabular}

TMERT1 restricted the error arc imaginary flow and increased the mean 5-minute coefficient of determination $\left(\mathrm{R}^{2}\right)$ between $\mathrm{TM}$ estimates and observations on a real network by $20 \%$ (Martin, 1997). Error arc upper bounds were equal to one-fortieth of the corresponding detected flow $\left(u_{j}(E)=0.025 * x_{j}(D)\right)$, rounded up. So, for 400 vehicles detected, corresponding error arcs could carry up to 10 unreal vehicles in each direction. Error arc flows were further discouraged by weighting error arcs three times more than all the other arcs. In this way, the large "through" TM flows fit up to $90 \%$ of observations, while small left and right TM estimates lacked reliability.

TMERT 2 applied the optimal weight constraints and produced a lower overall 5-minute Root Mean Square Error RMSE
(7 vehicles) than TMERT1 (10 vehicles) on the real network. (Karapetrovic and Martin, 2020.) The optimal weights are: 35 to the left $\left(w_{j}(T M-l)=35\right), 2$ to the through $\left(w_{j}(T M-t)=2\right)$ , 28 to the right $\left(w_{j}(T M-r)=28\right)$ TMs and 1 to all other $\operatorname{arcs}\left(w_{j}(D)=w_{j}(N D)=w_{j}(E)=1\right)$.

The latest model version, TMERT3, imposed the optimal lower bound constraints, producing a lower overall 5-minute RMSE (5 vehicles) than TMERT2 (7 vehicles) when applied to the real network. (Karapetrovic and Martin, 2021.) The optimal lower bound constraint regime entails iteratively lowering non-detector and TM arc lower bounds from three to reach feasibility $\left(l_{j}(N D)=l_{j}(T M)=\max \left\{[0,3] \mid x_{j} \in \Omega\right\}\right)$. Table 2 summarizes the TMERT 3 model version flow constraints for each arc type. 
Table 2

TMERT3 Constraints

\begin{tabular}{|c|c|c|c|c|}
\hline Arc Type & Flow & Lower Bound $\left(\mathbf{l}_{\mathrm{i}}\right)$ & Upper Bound ( $\left.\mathbf{u}_{\mathrm{i}}\right)$ & Weight $\left(w_{i}\right)$ \\
\hline Detector & $x_{j}(D)$ & $l_{j}(D)=x_{j}(D)$ & $u_{j}(D)=x_{j}(D)$ & $w_{j}(D)=1$ \\
\hline Non-detector & $x_{j}(N D)$ & $l_{j}(N D)=\max \left\{[0,3] \mid x_{j} \in \Omega\right\}^{(3)}$ & $\begin{array}{c}u_{j}(N D) \\
\text { (HCM estimate procedure) }\end{array}$ & $w_{j}(N D)=1$ \\
\hline Error & $x_{j}(E)$ & $l_{j}(E)=0$ & $u_{j}(E)=0.025^{(1)} * x_{j}(D)$ & $w_{j}(E)=1$ \\
\hline TM & $x_{j}(T M)$ & $l_{j}(T M)=\max \left\{[0,3] \mid x_{j} \in \Omega\right\}^{(3)}$ & $\begin{array}{c}u_{j}(T M) \\
\text { (HCM estimate procedure) }\end{array}$ & $\left\{\begin{array}{c}w_{j}(T M-l)=35^{(2)} \\
w_{j}(T M-t)=2 \\
w_{j}(T M-r)=28\end{array}\right.$ \\
\hline \multicolumn{5}{|c|}{ Added in: (1) TMERT1, (2) TMERT2, (3) TMERT3 } \\
\hline
\end{tabular}

\subsection{The Research Need}

Although shown to accurately estimate TM flows, the TMERT3 model version needs to present robustness to be practical. A robust model produces a similar predictive accuracy under changed assumptions. Extended validation tests TMERT3's accuracy when three assumptions are combined: detectors massively fail, a network is non-recurrently congested, and a network is more extensive with a higher flow to capacity $(\mathrm{v} / \mathrm{c})$ ratio.

First, TMERT3 estimates TMs from $15 \%$ of network flows detected. Inductive loops have relatively high malfunction rates and can fail to detect mid-block flow (Martin et al., 2003). For example, a quarter of 15,000 detectors in New York State in the 1980s were inoperative at any given time (Bikowitz and Ross, 1985; Klein et al., 2006). Since then, the number of malfunctioning detectors decreased by enhanced installation techniques and vigilant inspection, but not eliminated (Klein et al., 2006). Maintenance of inductive loops, placed underground, can be time-consuming. So, TM estimates can worsen due to an additional cut in the model input.

Second, all TMERT model versions always test peak hour traffic. However, peak hour traffic congestion is recurrent and generally predictable, so management strategies are often already established. Over half of the congestion is due to non-recurring events, such as traffic incidents, bad weather, work zones, or special events. (Rakha and Tawfik, 2009.) Congestion due to non-recurring events is typically erratic, having an unpredictable location, duration, and consequences (CSI, 2005). Therefore, the accuracy of TM estimates can deteriorate when traffic demand accumulates quickly and unexpectedly.

Third, TMERT3 has only been applied to small networks with a relatively low average network flow to capacity ratio (v/c). The largest network had 11 intersections with a $\mathrm{v} / \mathrm{c}$ ratio of up to $30 \%$. A more extensive, better-connected network offering more alternative routes could supplement the lack of mid-block detectors in estimating TMs with TMERT3. Moreover, TMERT3 is a quasi-dynamic estimator, so the demand during the modeling interval is constant but can change with the onset of the subsequent interval. Still, TMERT3 cannot model vehicles that remained in the network from the previous modeling interval. A high v/c rate can cause unstable traffic and queues formed at intersections, which can be impossible for TMERT3 to model. 


\subsection{Goal and Objectives}

The goal is to validate the TMERT3 model's robustness to a widespread midblock detector failure on a non-recurrently congested larger (over 20-intersection) urban network by showing it preserves predictive accuracy in estimating 5-minute TM flows from sparse detector data. The objectives addressing the goal are as follows:

- Examine predictive accuracy (Root Mean Square Error- RMSE) of TMERT3 in estimating 5-minute TM flows on a more extensive congested network when $15 \%$ of network flows are detected;

- Examine predictive accuracy (RMSE) of TMERT 3 in estimating TM flows on the same network using the same intervals when $20 \%$ of detectors fail, leaving $12 \%$ of network flows detected;
- Compare the overall predictive accuracy of TMERT 3 with $15 \%$ and $12 \%$ of network flows detected through 5-minute RMSE.

\section{Methodology}

The TMERT 3 model's robustness to a widespread mid-block detector failure is tested on the Orem/Provo, Utah, USA network (Figure 2). Orem and Provo are adjacent cities, populated by about 100,000 people each, and located $60 \mathrm{~km}$ south of Salt Lake City (US Census Bureau, 2020). The network holds a freeway, principal arterials, minor arterials, and urban collectors. $800 \mathrm{~N}$ bounds the study area in the north, University Parkway in the south, Geneva Road in the west, and University Avenue in the east.

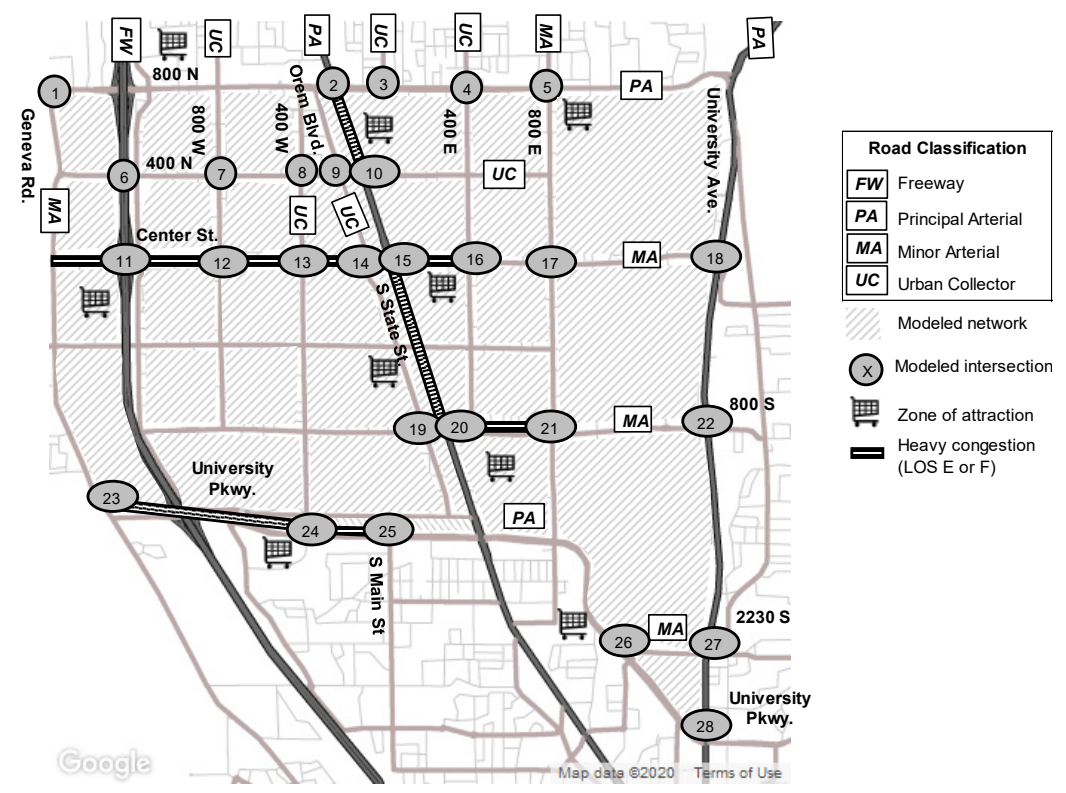

Fig. 2.

Orem/Provo, Utah, USA Study Area

Source: (Google Maps, 2020) 
The Orem/Provo network encompasses twenty-eight 4-legged signalized intersections, with 304 nodes and 495 nonerror arcs. The network is modeled twice for two detector coverage (DC) settings. The first setting (DC1) models seventy-four flows (15\%) as detected, so 148 error arcs move the imaginary flow, forming 643 arcs in total. The second setting (DC2) removes the fifth of the detectors, leaving 58 network flows detected (12\%), 116 error arcs, and a total of 611 arcs.

Twelve 5-min periods (1:10- 2:10 p.m.) for Friday, December 21, 2018, capture nonrecurrent congestion due to the Christmas shopping season. The flow to capacity (v/c) ratio on the analyzed network is $41 \%$, reaching $60 \%$. Over $11 \%$ of the network is heavily congested, operating with a Level of Service either E or F. However, the remaining network arcs are potential alternative routes, with relatively stable traffic flow and spare capacity. The overall network is not overcongested, as most of the formed queues clear by the end of a 5-minute modeling period.

The Automated Traffic Signal Performance Measures (ATSPM) website, hosted by the Utah Department of Transportation, provides 5-minute detector flows, signal settings, external loadings, and TM counts (UDOT, 2020). Google Maps deliver lane counts, turning options, and intersection design needed for HCM capacity estimation equations. TM counts are used as ground truth when assessing the accuracy of TM estimates delivered with TMERT3.
The Root Mean Square Error (RMSE) measures the squared difference between modeled and observed TMs. Lower RMSE implies that TM estimates deviate less from the observations. (Walther and Moore, 2005.) RMSE is convenient as it quantifies estimation error within the same scale (units) as a variable (Eq. 5).

$\operatorname{RMSE}($ vehicles $)=\sqrt{\sum \frac{\left(\hat{\mathrm{y}}_{\mathrm{i}}-\mathrm{y}_{\mathrm{i}}\right)^{2}}{\mathrm{~N}}}, \forall \mathrm{i} \in[1, \mathrm{~N}]$

Where: $y_{i}$ - observed TM flow, $\hat{y}_{i}$ - modeled TM flow, N- number of observations

Fewer detections require more flows to be estimated, possibly decreasing the accuracy and consistency of estimates. So, the comparison between RMSE values for two detector coverage settings presents TMERT3's robustness to a widespread detector failure.

\section{Results}

Calculated Root Mean Square Error (RMSE) between left, through, right, and overall $\mathrm{TM}$ estimates and the corresponding observations compared two detector coverage (DC) settings. The first setting (DC1) detected $15 \%$ of network arc flows. The second setting (DC2) arbitrarily removed $20 \%$ of the detectors, leaving $12 \%$ of network arc flows known. Table 3 shows RMSE calculated for both DC settings, accompanied by the mean, standard deviation (SD), minimum, and maximum values. 
Table 3

Calculated RMSE (veh.) for DC1 and DC2 for Left, through, Right, and Overall TMs

\begin{tabular}{|c|c|c|c|c|c|c|c|c|}
\hline RMSE (veh.) & \multicolumn{2}{|c|}{ Left } & \multicolumn{2}{c|}{ Through } & \multicolumn{2}{c|}{ Right } & \multicolumn{2}{c|}{ Overall } \\
\hline Period & DC1 & DC2 & DC1 & DC2 & DC1 & DC2 & DC1 & DC2 \\
\hline 1 & 25 & 25 & 33 & 34 & 17 & 18 & 26 & 27 \\
\hline 2 & 31 & 31 & 35 & 35 & 19 & 20 & 29 & 29 \\
\hline 3 & 27 & 27 & 35 & 36 & 15 & 16 & 27 & 28 \\
\hline 4 & 29 & 29 & 34 & 34 & 17 & 18 & 28 & 28 \\
\hline 5 & 29 & 29 & 37 & 37 & 19 & 19 & 29 & 29 \\
\hline 6 & 30 & 31 & 37 & 36 & 22 & 22 & 30 & 30 \\
\hline 7 & 21 & 21 & 27 & 26 & 13 & 14 & 21 & 21 \\
\hline 8 & 20 & 21 & 27 & 26 & 14 & 14 & 21 & 21 \\
\hline 9 & 19 & 19 & 25 & 25 & 12 & 14 & 19 & 20 \\
\hline 10 & 25 & 25 & 31 & 31 & 16 & 17 & 25 & 25 \\
\hline 11 & 27 & 28 & 34 & 34 & 17 & 18 & 27 & 27 \\
\hline 12 & 29 & 30 & 31 & 31 & 17 & 19 & 26 & 27 \\
\hline Mean \pm SD & $26 \pm 4$ & $26 \pm 4$ & $32 \pm 4$ & $32 \pm 4$ & $17 \pm 3$ & $17 \pm 2$ & $26 \pm 3$ & $26 \pm 3$ \\
\hline Min-Max & $19-31$ & $19-31$ & $25-37$ & $25-37$ & $12-22$ & $14-22$ & $19-30$ & $20-30$ \\
\hline
\end{tabular}

The mean RMSE was $26 \pm 4$ vehicles for both DC settings, ranging from 19-31 vehicles for left TMs. Similarly, through TM estimateobservation pairs yielded a mean RMSE of 32 \pm , ranging from 25-37, for both DC1 and DC2 settings. Right TMs were estimated with the mean RMSE of $17 \pm 3$ with a minimum of 12 and a maximum of 22 for DC1. The detector removal produced slightly more consistent RMSE $17 \pm 2$, ranging between 14 and 22 . The mean RMSE for overall TMs was $26 \pm 3$ for both DC settings. However, overall TMs were more consistently estimated when a fifth of the detectors fails, as the RMSE range narrowed from 19-30 to 20-30.

\section{Discussion}

The 28-intersection Orem/Provo, Utah network is used to test TMERT3's accuracy in estimating twelve 5-minute TMs during non-recurrent congestion due to seasonal Christmas shopping. Initially, $15 \%$ of the network flows are detected. Then, $20 \%$ of the initial detectors are removed, leaving less than $12 \%$ of network flows known. The Root Mean Square Error (RMSE) between observed and estimated TMs is calculated for two detector coverage settings and compared. The main research findings are as follows:

1. TMERT3 produces an overall 5-minute RMSE of 26 vehicles when applied to a real and more extensive congested network, with $15 \%$ of network flows detected.

2. TMERT3 yields an overall 5-minute RMSE of 26 vehicles when applied to the same network over the same period when $12 \%$ of network flows are detected.

3. TMERT3 produces the same overall 5-minute RMSE when $15 \%$ and $12 \%$ of network flows are detected (26 vehicles).

Therefore, the results indicate that eliminating flow detectors does not diminish estimation accuracy. TMERT3 has the same predictive accuracy (RMSE $=26$ vehicles) when $15 \%$ and $12 \%$ of network flows are detected when applied to the 
urban congested, more extensive network, presenting robustness to a widespread detector failure.

TMERT3 consistently performs better when estimating dominant flows. Almost all through TMs match the observations $\left(\mathrm{RMSE}=22, \mathrm{R}^{2}=95 \%\right)$ on a real network with $20 \%$ of failed detectors. The mean 5-minute through TMs are 92, with a maximum of 576. On the other hand, left and right TMs are small. Right TMs have a mean of 18 , the maximum flow 138, and yield RMSE of 11 and $\mathrm{R}^{2}$ of $30 \%$. It is even worse for left turns, as RMSE is 15 and $\mathrm{R}^{2}$ is only $28 \%$. The mean number of left-turning vehicles is 24 , with a maximum of 132 .
The average network intersection proportion is $18 \%: 69 \%: 13 \%$, so typically, $18 \%$ of vehicles turn left at the intersection approach and $13 \%$ turn right. Although more vehicles turn left than right, left turns are estimated less accurately. The reason for reduced estimation accuracy could be due to U-turning vehicles modeled as left TMs. So, turnaround vehicles can appear on the incorrect outflow link, causing flow disparity on the entire network. Left TMs estimation accuracy can significantly reduce with the increase in U-turns and the number of failed detectors. Figure 3 shows how TM observations and estimates compare during a typical observation period, from 1:55-2:00 p.m.
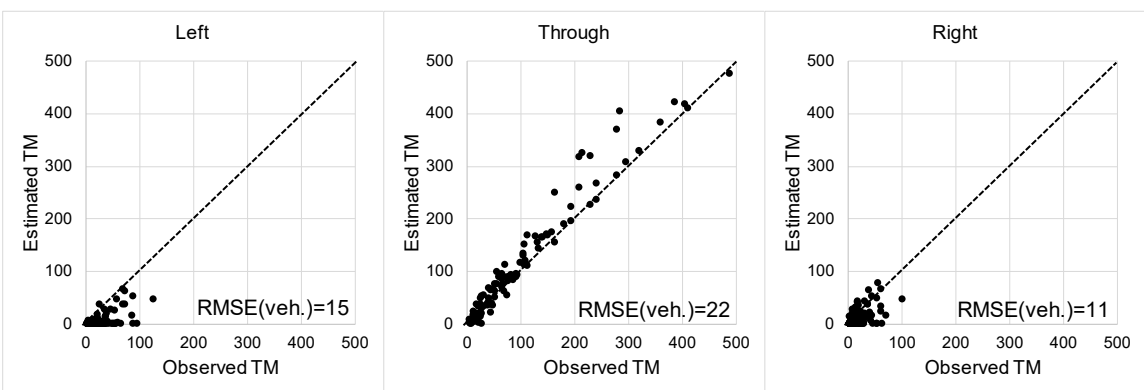

Fig. 3.

Comparison of Estimated and Observed TM Flows for DC2 (the Orem/Provo, Utah Network, 1:55 p.m.-2:00 p.m.)

Underestimating low flows can be attributed to changed driver behavior on congested streets, as more vehicles turn seeking alternative routes. A headway is smaller, allowing additional vehicles to turn during the same signal phase. However, TMERT3 fails to increase its upper arc bounds to adapt to the changed driving regime. So, TMERT 3 can falsely assign flow to through instead of to the left or right TM arcs due to rigid limits. As a result, left and right TMs can be underestimated and through TMs overestimated.
Although shown to accurately estimate TMs and be robust to a widespread detector failure, TMERT 3 can be further examined and improved. Future work should express the acceptable detector failure rates as a function of the detector configuration and the detected flow. Also, TM upper bounds should be presented as a function of congestion to improve TM estimation accuracy.

The average network flow to capacity ratio (v/c) on the analyzed network is $41 \%$ and 
up to $60 \%$. The predictive accuracy of TMERT 3 could decrease with the severity of congestion, as TMERT 3 cannot model traffic extremely unstable or close to a breakdown for long periods. So, future research should identify the $\mathrm{v} / \mathrm{c}$ ratio associated with a TMERT3 failure.

The research on the optimal size and shape of a modeled network should provide guidelines for using the TMERT 3 model version in estimating intersection demand. Further study should simulate an incident to verify the effect of diverting traffic to TMERT3 -identified network routes with spare capacity and comparing TMERT3's performance to other available low-cost operational treatments, such as remedial signal plan change.

\section{Conclusion}

TMERT3 can instantly produce 5-minute TM estimates and identify feasible alternative routes on the congested network. Here, TMERT3 presented robustness to a widespread detector failure on a congested, more extensive network (over 20 intersections). So, TMERT 3 can consistently provide traffic demand information, despite $20 \%$ of network mid-block detectors simultaneously failing. Therefore, TMERT3 is applicable and efficient in minimizing traffic delays, vehicle operating expenses, and traveler frustration.

\section{References}

Bikowitz, E. W.; Ross, S. P. 1985. Evaluation and Improvement of Inductive Loop Detectors., Transportation Research Record: Journal of the Transportation Research Board 1010: 76-80.
Chen, A.; Chootinan, P.; Ryu, S.; Lee, M.; Recker, W. 2012. An intersection turning movement estimation procedure based on path flow estimator, Journal of Advanced Transportation 46(2): 161-176.

CSI. 2005. Traffic Congestion and Reliability Trends and Advanced Strategies for Congestion Mitigation. Cambridge Systematics Inc., Texas Transportation Institute. $140 \mathrm{p}$.

Ghods, A.H.; Fu, L. 2014. Real-time estimation of turning movement counts at signalized intersections using signal phase information, Transportation Research Part C: Emerging Technologies 47: 128-138.

Google Maps. 2020. Orem/Provo, Utah, USA. Available from Internet: <https://www.google.com/ maps/@40.2819374,-111.6987692,14z>.

Gordon, R.L.; Warren, T. 2005. Traffic Control Systems Handbook. Technical Report No. FHWA-HOP-06-006. Washington, DC. 369p.

HCM 2010. Highway Capacity Manual. Transportation Research Board. Washington, D.C., USA. 1,475p.

Karapetrovic, J.; Martin, P. T. 2020. Estimating intersection turning movement flows with a NETFLO algorithm: weight constraint calibration, Advances in Transportation Studies, an International Journal 52: 73-88.

Karapetrovic, J.; Martin, P. T. 2021. The Turning Movement Estimation In Real-Time (TMERT) Model: Lower Bound Constraint Calibration. In Proceedings of the 12th International Conference on Ambient Systems, Networks and Technologies (ANT 2021), Warsaw, Poland (in print).

Kennington, J.L.; Helgason, R.V. 1980. Algorithms for Network Programming. New York: A Wiley-Interscience Publication, New York, USA. 291p. 
Klein, L.A.; Mills, M.K.; Gibson, D.R.P. 2006. Traffic Detector Handbook. Third edition, Volume I, US Department of Transportation, Federal Highway Administrationdoi: FHWA-HRT-06-108 October. 291p.

Lan, C.J. 2001. Adaptive turning flow estimation based on incomplete detector information for advanced traffic management. In Proceedings of the IEEE Conference on Intelligent Transportation Systems - ITSC, 830-835.

Leard, B.; Linn, J.; Munnings, C. 2019. Explaining the Evolution of Passenger Vehicle Miles Traveled in the United States, The Energy Journal 40(1): 25-54.

Martin, P. T. 1997. Turning Movement Estimation in Real-Time, Journal of Transportation Engineering 123(4): 252-260.

Martin, P. T.; Feng, Y.; Wang, X. 2003. Detector Technology Evaluation. Report No. MPC Report No. 03-154. Fargo, ND. 140p.

Mirchandani, P.B.; Nobe, S.A.; Wu, W.W. 2001. Online Turning Proportion Estimation in Real-Time TrafficAdaptive Signal Control, Transportation Research Record: Journal of the Transportation Research Board 1748(1): 80-86.

Mozolin, M.; Thill, J.C.; Usery, E.L. 2000. Trip distribution forecasting with multilayer perceptron neural networks: A critical evaluation, Transportation Research Part B: Methodological 34(1): 53-73.

Nakatsuji ,T.; Nakano, K.; Nanthawichit, C.; Suzuki, H. 2004. Estimation of Turning Movements at Intersections: Joint Trip Distribution and Traffic Assignment Program Combined with a Genetic Algorithm, Transportation Research Record: Journal of the Transportation Research Board 1882(1): 53-60.

NCHRP. 2008. Task 63: Effective Strategies for Congestion Management, National Cooperative Highway Research Program (NCHRP) Project 20-24. Cambridge Systematics, Inc. Cambridge, MA. 214p.
Rakha, H.; Tawfik, A. 2009. Traffic Networks: Dynamic Traffic Routing, Assignment, and Assessment. Report No. FHWA-HOP-09-015. Washington, DC: US Department of Transportation, Federal Highway Administration. 22 p.

Rao, A.M.; Rao, K.R. 2012. Measuring urban traffic congestion-a review, International Journal for Traffic \& Transport Engineering 2(4): 286-305.

Riouali, Y.; Benhlima, L.; Bah, S. 2019. An Integrated Turning Movements Estimation to Petri Net Based Road Traffic Modeling, Journal of Sensor and Actuator Networks 8(3): 1-18.

Schrank, D.; Eisele, B.; Lomax, T. 2019. 2019 Urban Mobility Report. Texas A\&M Transportation Institute, The Texas A\&M University System. 50p.

Shirazi, M.S.; Morris, B.T. 2016. Vision-Based Turning Movement Monitoring: Count, Speed \& Waiting Time Estimation, IEEE Intelligent Transportation Systems Magazine 8(1): 23-34.

Tuydes-Yaman, H.; Altintasi, O.; Sendil, N. 2015. Better estimation of origin-destination matrix using automated intersection movement count data, Canadian Journal of Civil Engineering 42(7): 490-502.

UDOT. 2020. AADT (Open Data) | UDOT Open Data. Utah Department of Transportation (UDOT). Available from Internet: <http://data-uplan.opendata.arcgis.com/ datasets/c2c6fe2c52b141b6afb4374d5825c611_0>. (Accessed: 17 August 2020).

US Census Bureau. 2020. QuickFacts: Provo City, Utah; Orem city, Utah; United States. Available from Internet: <https://www.census.gov/quickfacts/fact/ table/provocityutah,oremcityutah,US/PST045219>. (Accessed: August 17 2020).

Vespa,J.; Medina, L.; Armstrong, D. 2020. Demographic turning points for the United States: Population 
projections for 2020 to 2060. Current Population Reports No. P25-1144. 15p.

Walther, B. A.; Moore, J. L. 2005. The concepts of bias, precision and accuracy, and their use in testing the performance of species richness estimators, with a literature review of estimator performance, Ecography 28(6): 815-829.

Wu, J. H.; Thnay, C. 2001. An O-D based method for estimating link and turning volume based on counts, ITE District 6: 8-11.
Yang, Z.; Pun-Cheng, L.S.C. 2017. Vehicle detection in intelligent transportation systems and its applications under varying environments: A review, Image and Vision Computing 69: 143-154.

Zhai, W.X.; Ardian, D. 2020. Traffic flow control of the intersection in urban traffic system under the environment of internet of vehicles, Advances in transportation studies (Special Issue 1): 31-40. 